\title{
The Application of Neural Network in the Evaluation of the Computer Network Security Zhenyou Sui
}

Inner Mongolia University for the Nationalities, Tongliao, 028043, China

\author{
Keywords: Computer network, Safety Evaluation, Neural Network
}

\begin{abstract}
Computer network technique, which plays an important role in dealing with file, official document, can also be applied inside the enterprise, outside the network and in the globalized internet. As the computer network provides so much convenience, it also faces many safety loopholes, such as the bug, virus, etc. Therefore, establishing the evaluation system of computer network safety can realize the instantaneity and safety of the evaluation. Neural network which is the most widely used technology in the evaluation of computer network safety is significant for the development of the computer network technology. This text discusses the neural network in the evaluation of computer network safety, analyzes the basic principle of neural network, the importance and application of neural network in the evaluation of the computer network safety.

With the constant development of scientific technology, the continuous increase of the socioeconomic level, computer network technology has got new development. With the constant application of computer, more and more factors can threat the safety of the computers and they appear in various forms such as hacker, virus and system bug, etc. These comparably complicated factors are connected. There exist non-linear relations between the evaluation factor of computer network safety and the result. The traditional safety evaluation system is difficult to operate, so the relation between the factor and the safety evaluation cannot be discovered accurately, which makes the accuracy of the evaluation not high. Since the professional safety evaluation system requires a lot of knowledge, then the evaluation result can not be accurate, objective and scientific with no scientificity. The neural network, which is the self-adapted and non-linear dynamic system formed by the connection of different nerve cells, have the effect of calculation, adaptation, identification and controlling. Applying the neural network in the computer network reasonably can improve the scientificity and efficiency of the safety evaluation.
\end{abstract}

\section{I .The Introduction and Basic Principle of Neural Network Technology}

With the constant progress of computer network, it has become an indispensable part in our life. People also depend more on the network. But at the same time, the problem of the computer network safety is becoming more obvious. In order to guarantee the safety of the computer network system, people started to study and analyze the neural network technology earlier in the 1940th and tried applying the neural network into the network safety. The development of neural network can mainly be divided into three stages: The first stage is the beginning period from 1947 to 1969 during which the rules and model of neural network was born; the second stage is the transition period from 1970 to 1986 during which the optimizing of the computing method and associative memory was born; the third stage is the development period from 1987 to the present during which the gradual tide of dotcom boom promoted the development of network technology.

In neural network, the neural network algorithm is one of the most important computing method and is also called Back Propagation Network which is composed of three parts including the hidden layer, the input layer and the output layer. In this network, some network nerve cells can only pass the different states to the next necessary network nerve cell unidirectionally. The process of transmission mainly includes the error message through backward propagation and the correct message through forward propagation. The basic operating principle is that the input message goes through the hidden layer gradually through forward propagation and then after some processing, it enters the output layer. But during the transmission process, if the desired valued hasn't been gained, then there will be wrong messages on the terminal of the nerve cell which eventually leads to the 
returning of the wrong messages to the input lays from the same route. After constant and repetitive process, the error is the minimal to guarantee the accuracy of the message.

\section{The Necessity of Neural Network in the Evaluation of Computer Network Safety}

(1). The Strong Self-adaptation of Neural Network

The neural network possesses good ability to adjust to the environment and high learning ability. If the neural network is in the input or output model, then the neural network can regulate itself to reduce the error. In some degree, it can train itself to conclude the rule in the transmission process.

(2). The Fault Tolerance of the Neural Network

Compared with the past model, the neural network has low sensitiveness on the level such as the information integrity and noise. The basic cause of the problem is that the neural network node can only reflect one characteristic, which means that it will not have big influence even when the noise or the information is not complete.

(3) The Linearity of the Neural Network

It costs the neural network a lot of time in the training. But once the training is completed, the working efficiency will be greatly improved. Neural network works very quickly when it acquires the transmission result, so it is very quick and convenient when it is applied to use. Therefore, the neural network can be linear.

(4). The Function of Extrapolation and Automatic Extraction Owned by the Neural Network

In the neural network training, it can gain the effect of promotion knowledge from the sample and develop into the whole sample. It can also be trained with direct data and value to confirm the relations between the result and the cause.

\section{The Application of Neural Network in the Evaluation of Computer Network Safety.}

(1) The Computer Network Safety

The safety of the computer network actually is to safeguard the integrity, workability and confidentiality of the data in the computer network by applying the control management measures and advanced scientific technology reasonably. The computer network safety mainly includes physical security and network security. Physical security is to protect the associated equipment of the computer with the use of physical property in case of the destruction and lose of the data. Logic security is actually the confidentiality, security, usability and integrity of the data information. The computer network security not only includes the reasonable control of the network hardware and software, but also the convenient service and resource sharing.

Computer network security has five features which are integrity, usability, confidentiality, auditing and controllability. Computer network is free, open and international, which makes the computer network suffer the attacks from many aspects, such as communication protocol attacks, transmission link attacks and software loophole attacks, etc. The computer network security faces more and more challenges. Not only the local network users can harm the network, also the users in other regions can attack and break it in some degree. At present stage, many computer networks do not have the technology to limit the users, so they can publicize and gain information freely.

(2). The Evaluation System of the Computer Network Security

The evaluation system of the computer network is the system founded due to the influence on the computer network security. The main factors which influence the computer network security can be reflected comprehensively, objectively, scientifically and reasonably through the evaluation system of computer network. The evaluation of computer network security requires an evaluation indicator. The indicator should be selected selectively by integrating many factors to reflect the evaluation information accurately. In the whole evaluation system of computer network security, the effect of neural network should be guaranteed to have better full development. The first-degree indicator in the evaluation system of the computer network security consists of three aspects including logic security, management security and physical security. Logic security indicator includes 9 second-degree indicators which are data recovery, intrusion precaution, software security, access 
control, data encryption, system auditing, backup data, anti-virus measures and digital signature. The indicators of the physical security includes 6 second-degree indicators which are equipment security, supply security, the security of the machine room, fault and redundancy toleration, electromagnetism safety and the line security. The system of the computer network security includes the following five establishment principles; The first one is feasibility. The evaluation system of the computer network security should have the corresponding appraisal for the convenient operation and evaluation of security system. The second one is independence. The evaluation system of computer network security should avoid the repetitive indicator and reduce the relations among the indicators to the largest extent to guarantee that it can solve the problems of network security. The third one is accuracy. The evaluation system of the computer network security should accord with all the indicators in the indicators to safeguard the secure technology. The fourth one is the completeness. The proper indicators should be selected in the evaluation system of the computer network security to reflect the main characters comprehensively and completely and to guarantee the accuracy and liability of the result. The fifth is conciseness. The evaluation system of the computer network security should have coherent, representative and brief indicators.

(2). The Value and Standardization of the Evaluation Indicator

Different factors can be described through different indicator which includes mainly two standards: qualitative assessment and quantitative assessment. They can reflect the different security situations of computer network through the different emphasis of different standards. Therefore, in real evaluation process, different value criterions should be conducted proper standardization aiming at the two different indicators. When the quantitative criterion is quantized, concrete analysis about the concrete reason should be processed due to the practical value situation of the computer network. Apart from that, there exists difference among the measurement units. In the process of standard quantification, generally the value range is between 0 and 1 . In the evaluation with qualitative indicators, the method of expert decision is adopted to conduct a reasonable evaluation toward the computer network system. The method should be dealt with standardization to carry out a rational compare with the quantitative standardization.

(3). The Establishment of the Evaluation Result, the Evaluation Collection

The evaluation of the safety evaluation result can be divided into four grades according to the indicators of the safety assessment and the real situation of the evaluation character. They are security, basic security, general security and much insecurity. The grade of security can tell that the computer network has high guarantee capability. The grade of basic security can tell that the computer network has certain guarantee capacity. The grade of general security can tell that the computer network has limited guarantee capacity. The grade of much insecurity can tell that the computer network has bad guarantee capacity with serious problems of security.

\section{The Design of the Neural Network's Evaluation Model of the Computer Network Security.}

At the present stage, BP Neural network is used the most widely with application prospect in neural network. Therefore, we should choose BP neural network as the specific objective for introduction. The basic pointcut of BP neural network is that the data error can be reduced after transmission training of the samples in alterative direction to reach the desired effect and be convenient for the practical application.

(1). The Design of the Input Layer

When the BP neural network is designed, the number of the node in the input layer has to be identical to the number of the evaluation criterion according to the stipulation. It can also be said that the number of the secondary-class indicators owned by the first-class indicators should be the amount of the neural nodes in the construction of the evaluation model of the neural network, so the number of the neural nodes can be corresponded to the labeled indicators.

(2). The Design of the Hidden Layer

In practical construction process, many BP neural networks employ the single hidden layer and the number of the hidden nodes will influence the property of the computer network in some degree. 
Therefore, it should be emphasized greatly. If there are too many selections about the quantity of the nodes in the hidden layer, then the study time can be increased, and even the probability of the error can be affected. Consequently, it will have some impact on the efficiency of computer network study; if there are too fewer selections about the number of the nodes in the hidden layer, the fault toleration and nonlinearity are influenced in some degree. So when the nodes in the hidden layer are selected, a reasonable choice should be made with the proper scale according to the past experience.

(3). The Design of the Output Layer

When the output layer is designed in the BP neural network, the evaluation result should be reflected fully based on the evaluation result, the evaluation collection. If the output layer in the BP neural network has two nodes, the result of the output can be assumed as $(1,1)$ indicating security $(1,0)$ indicating basic security $(0,1)$ indicating general security $(0,0)$ indicating much insecurity

(4). The Study of the Computer Network Security Evaluation Model of the Neural Network

When the evaluation model of BP neural network is constructed, every layer should have the weight to start the connection, which means to conduct reasonable study during the construction of the BP neural network evaluation model. Through the study, the difference between the evaluation result and the real needs should be reduced to the largest extent to increase the percent of contact area.

(5). The Verification of the Computer Network Security Evaluation Model of the Neural Network.

For the evaluation model of BP neural network, it requires not only reasonable study and design, but also should be verified to guarantee the feasibility of the model. A series of data should be selected reasonably to be verified. First of all, some sample information should be input to carry out the evaluation of the neural network. If the evaluation result confirms to the expected values designed, then it shows that the established model of neural network is accurate.

\section{Conclusion}

In total, with the progress of scientific technology, the neural network technology has gained rapid development and is applied in more areas gradually. The application of neural network technology in the evaluation of computer network security is also pretty extensive and has made some achievements. The main advantages of the application of neural network in computer network are: it possesses higher evaluation authenticity, objectivity and liability which lay a solid foundation for the development of computer network. Although the neural network technology has gained some achievements, it needs further development, the greatest promotion in the digitalization of social economy, and the comprehensive development of informatization and intelligence. All of these provide protection for the development in our country.

\section{References}

[1] Geng Zhonghua.The Application Study of the Neural Network in the Evaluation of Computer Network Security[J].Network Security Technology and Application, 2014(9):87-88.

[2] Liutao. The Study of the Application of the Neural Network in the Evaluation of Computer Network Security[J]. E-journal during the Education Era(Teacher Edition), 2014(27):113.

[3] Wu Renjie. The Study of the Application of the Neural Network in the Evaluation of Computer Network Security[J] The Computer Stimulation,2011,28(11):126-129.

[4] Zhenggang. The Application Study of the Neural Network in the Evaluation of Computer Network Security[J], Network Security Technology and Application,2014(9):55,57.

[5] Guo Lijuan. The Application of the Neural Network in the Evaluation of Computer Network Security[J]. The Value Engineering, 2012,31(21):187-189. 